\title{
A Simple Multichromophore Design for Energy Transfer in Distyrylbenzenes with Pyrene Pendants
}

\author{
Cesar A. Sierra and Paul M. Lahti* \\ Department of Chemistry, University of Massachusetts, Amherst, MA 01003 USA \\ E-mail: lahti@chem.umass.edu
}

Figure S1. Qualitative overlap of pyrene emission spectrum and core (11) absorption spectrum p S1

Figure S2. Excitation spectra for 11, 1:2 mixture of 11 with pyrene, and 1-5. p S2

Figure S3. Comparisons of UV-vis and excitation spectra for $\mathbf{1}$ and 2. p S3

Figure S4. Solvent effect on luminescence of physical mixtures of pyrene with $11 . \quad$ p S4

Figure S5. Solvent effect on luminescence of model compound 19. p S4

Figure S6. Molecular mechanics optimized structure of 1 p S5

Figure S7. 2D NOESY spectrum of 1 in $\mathrm{CDCl}_{3}$ with a D8 time of $500 \mathrm{~ms}$ p S6

$\begin{array}{ll}\text { Synthetic details. } & \text { p S7 }\end{array}$

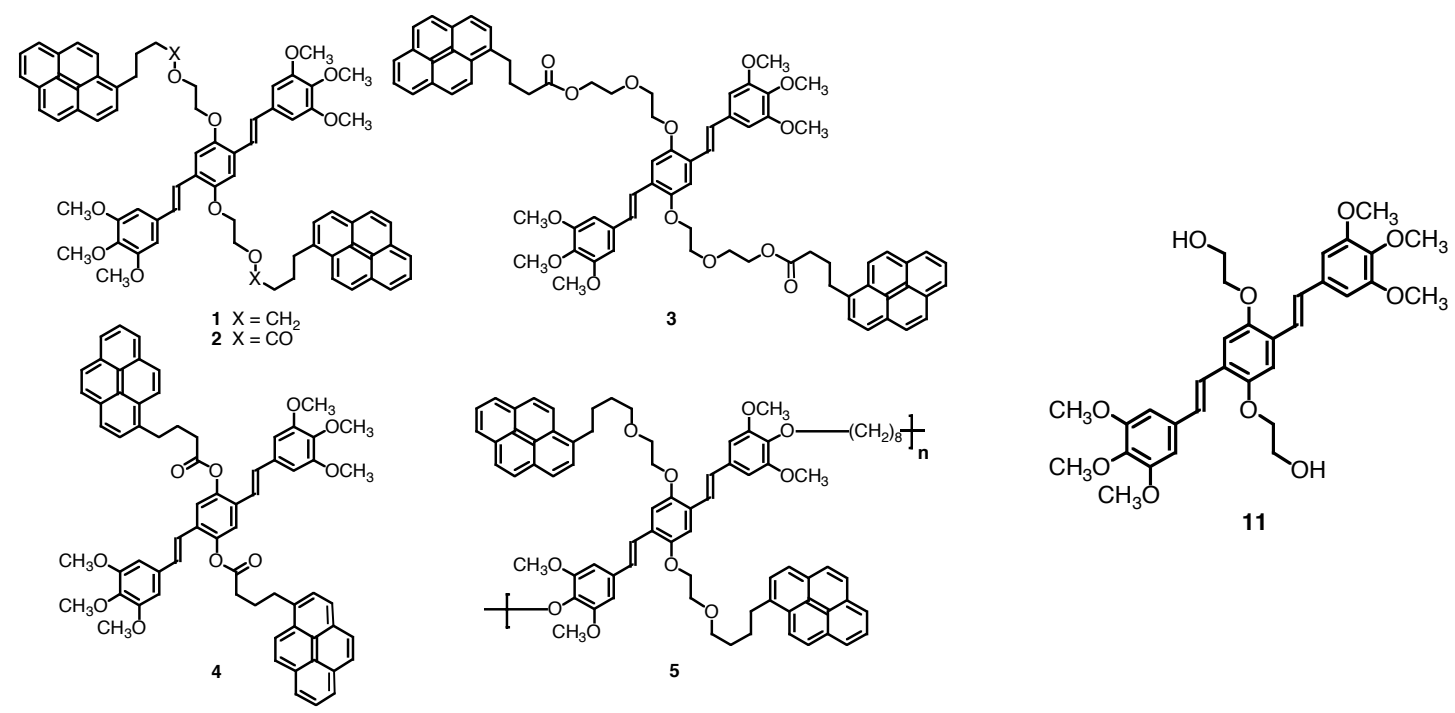


Figure S1. Qualitative overlap of pyrene emission spectrum and core (11) absorption spectrum shows good overlap of pyrene emission with core absorption.
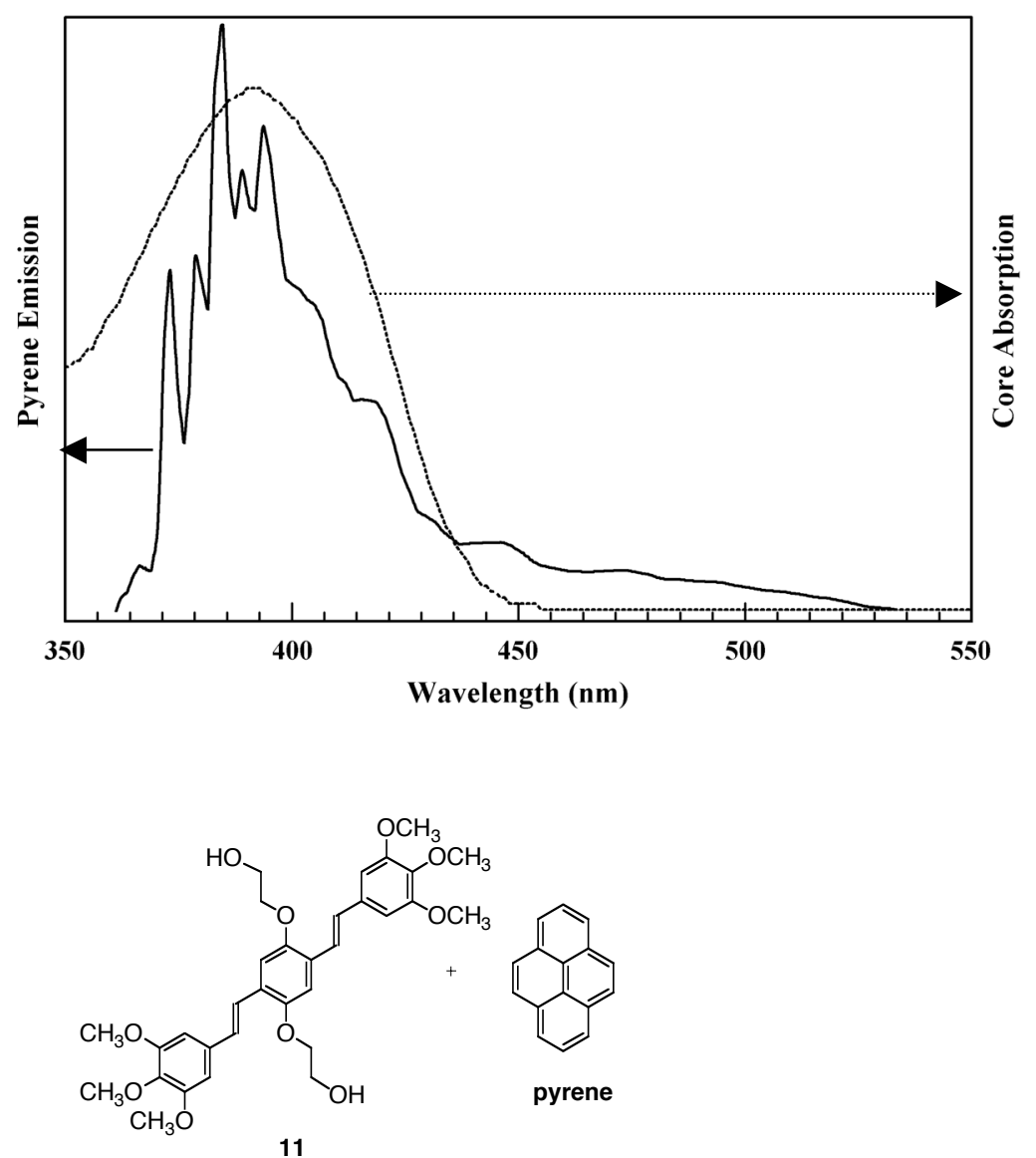
S2

Figure S2. Excitation spectra for 11, 1:2 mixture of $\mathbf{1 1}$ with pyrene, and 1-5. All spectra in chloroform at room temperature at about 1 micromolar concentrations, all spectra monitored at $445 \mathrm{~nm}$ (core component emission tail).

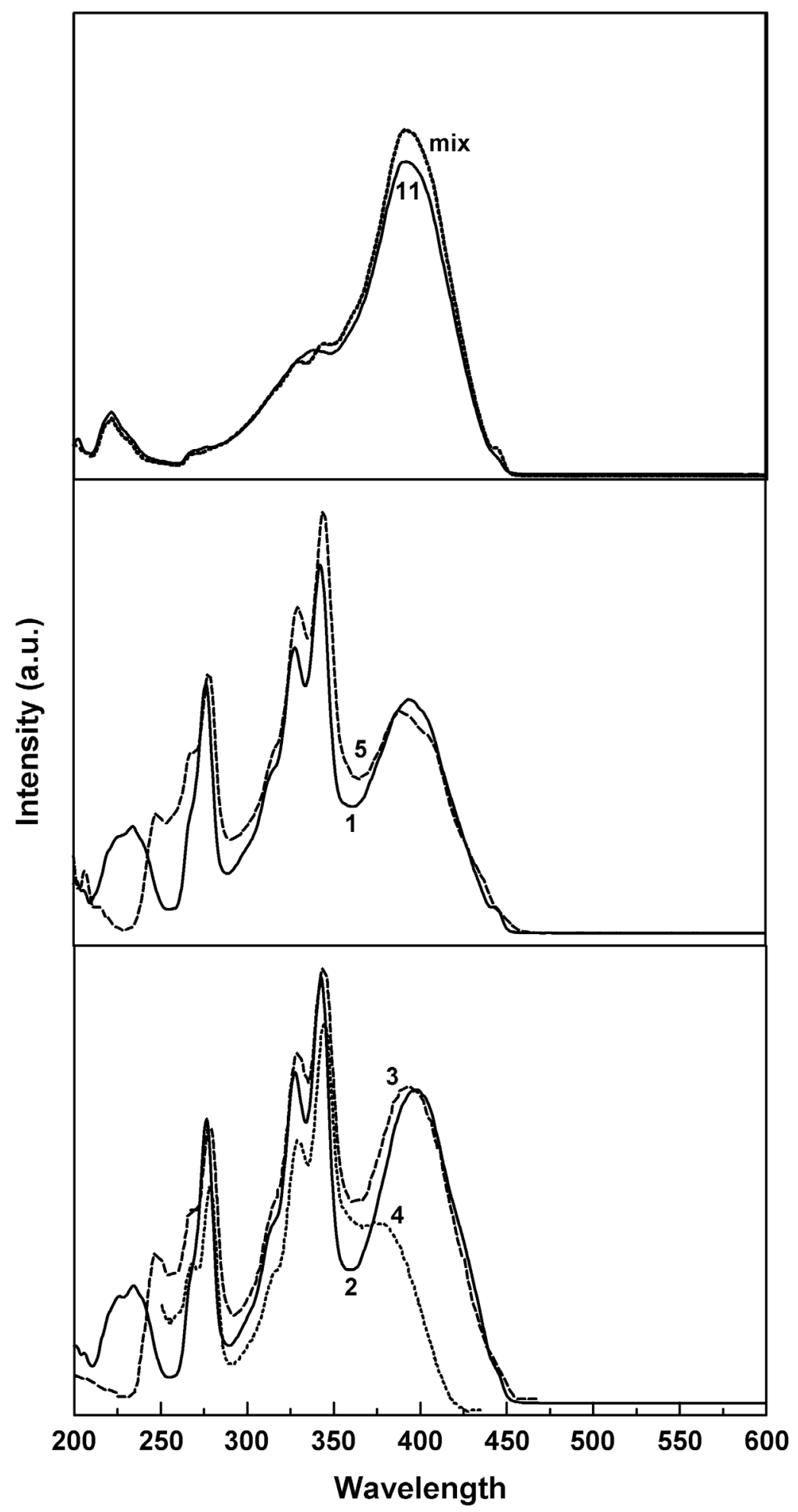


Figure S3. Example comparisons of UV-vis (solid lines) and excitation spectra (dashed lines) in chloroform for $\mathbf{1}$ and 2, normalized on the maxima of the long wavelength peaks at about 400 $\mathrm{nm}$. Excitation spectra monitored at $445 \mathrm{~nm}$. The normalized ratio Area(excit)/Area(UV) $=\eta_{\mathrm{ET}}$, the energy transfer efficiency.
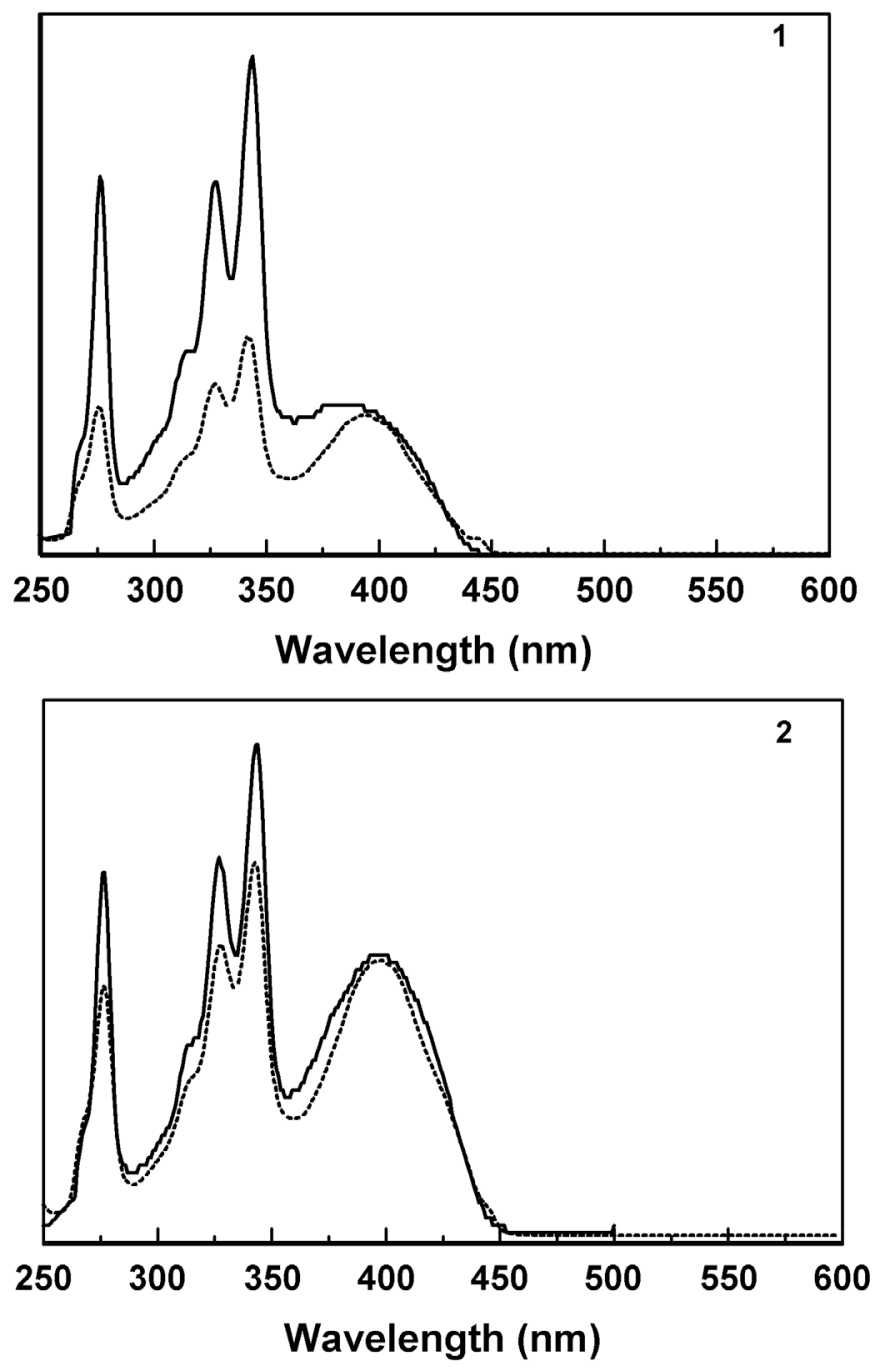
Figure S4. Solvent effect on luminescence of physical mixtures of pyrene with 11. Solid line in chloroform, broken line in tetrahydrofuran; both spectra obtained with $276 \mathrm{~nm}$ excitation.

Ordinate scales are normalized for the spectra relative to one another.

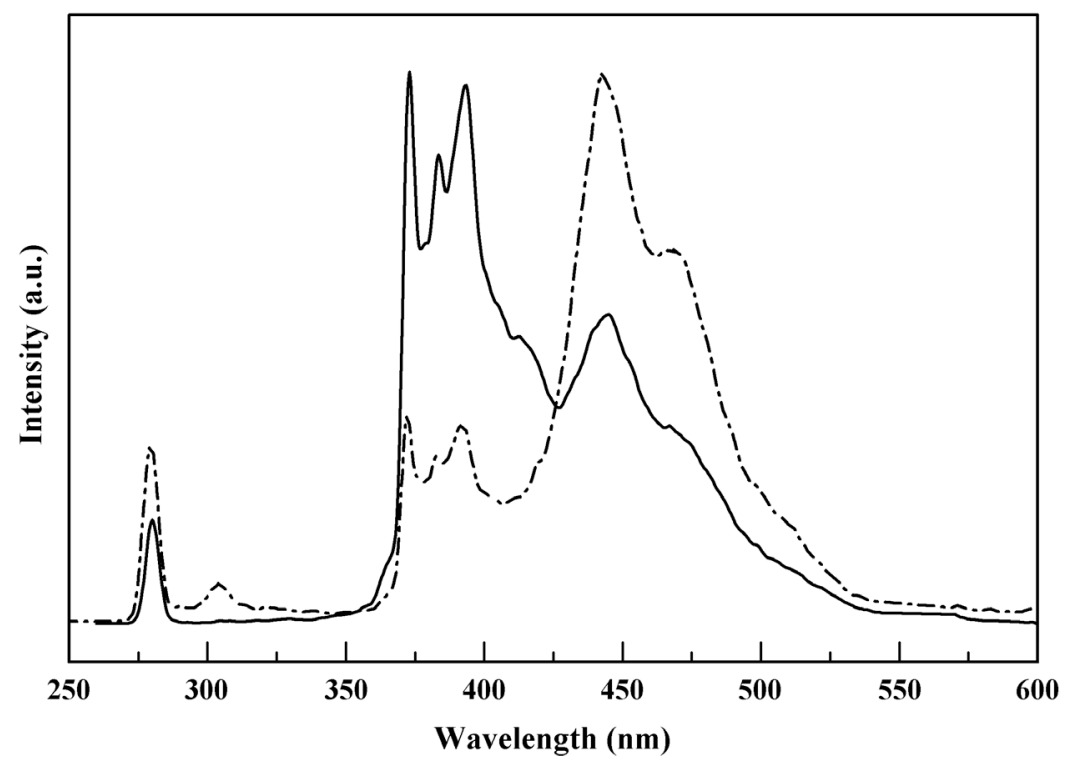

Figure S5. Solvent effect on luminescence of model compound 19. Spectra obtained with 365 $\mathrm{nm}$ excitation. Ordinate scales are normalized for the spectra relative to one another.

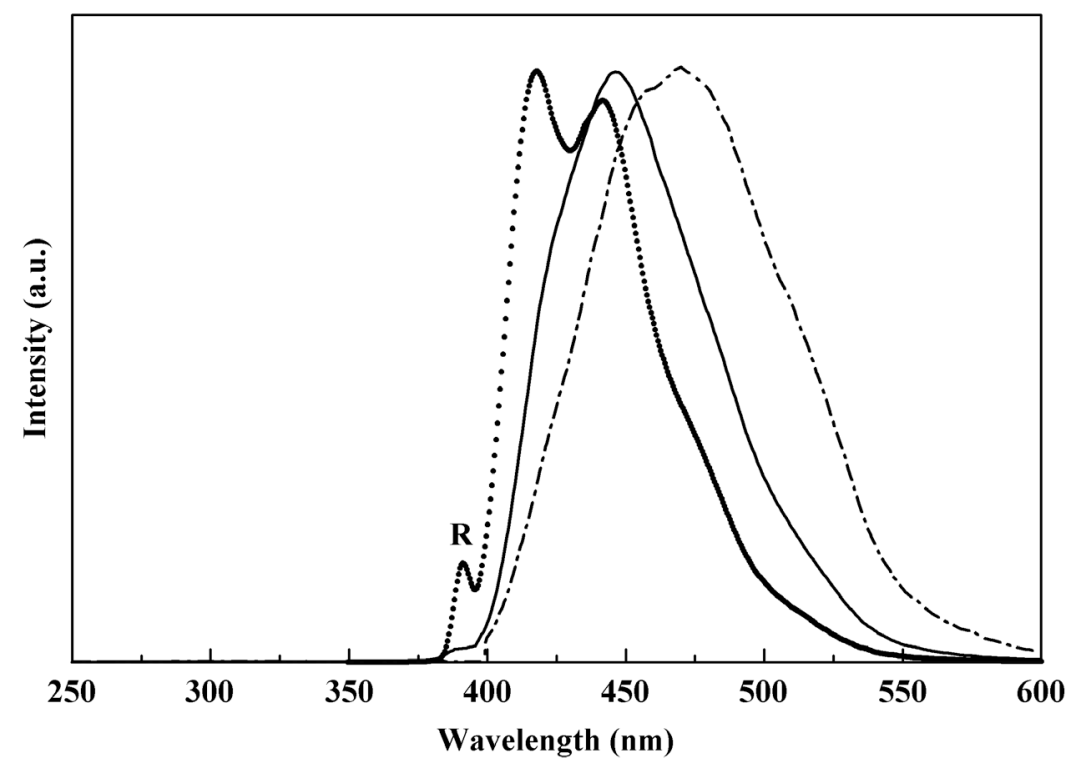


Figure S6. Molecular mechanics optimized structure of 1. Computations were carried out using the UFF force field (Rappe, A. K.; Casewit, C. J.; Colwell, K. S.; Goddard, W. A., III; Skiff, W.M. J. Am. Chem. Soc, 1992, 114, 10024 within the Cerius ${ }^{2}$ suite of programs by Accelrys (version 3.8 for SGI computer using Irix 6.5.x, (1998)).

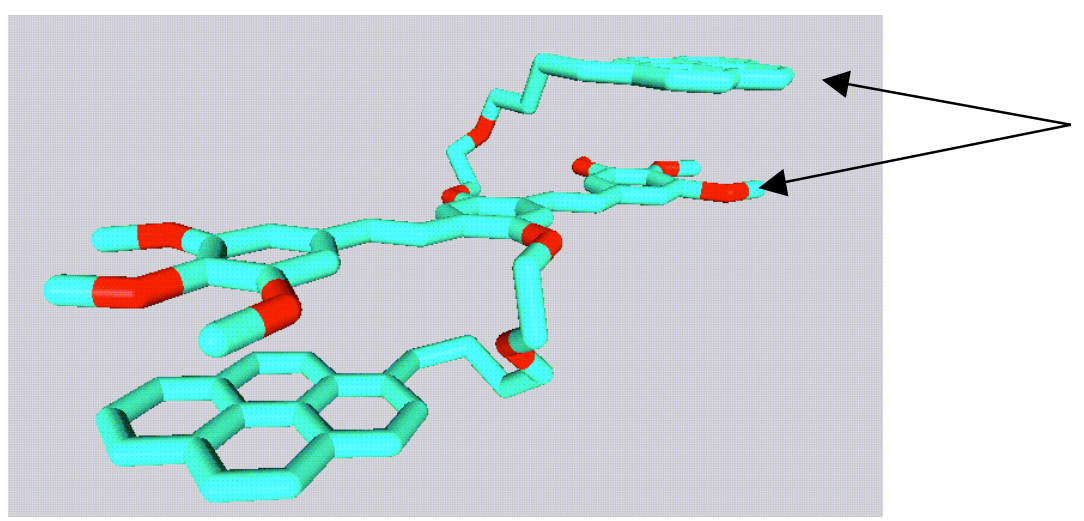

Distance of pyrene to terminal trimethoxyphenyl ring is about 3.5 angs. 
Figure S7. 2D NOESY spectrum of 1 in $\mathrm{CDCl}_{3}$ with a D8 time of $500 \mathrm{~ms}$..
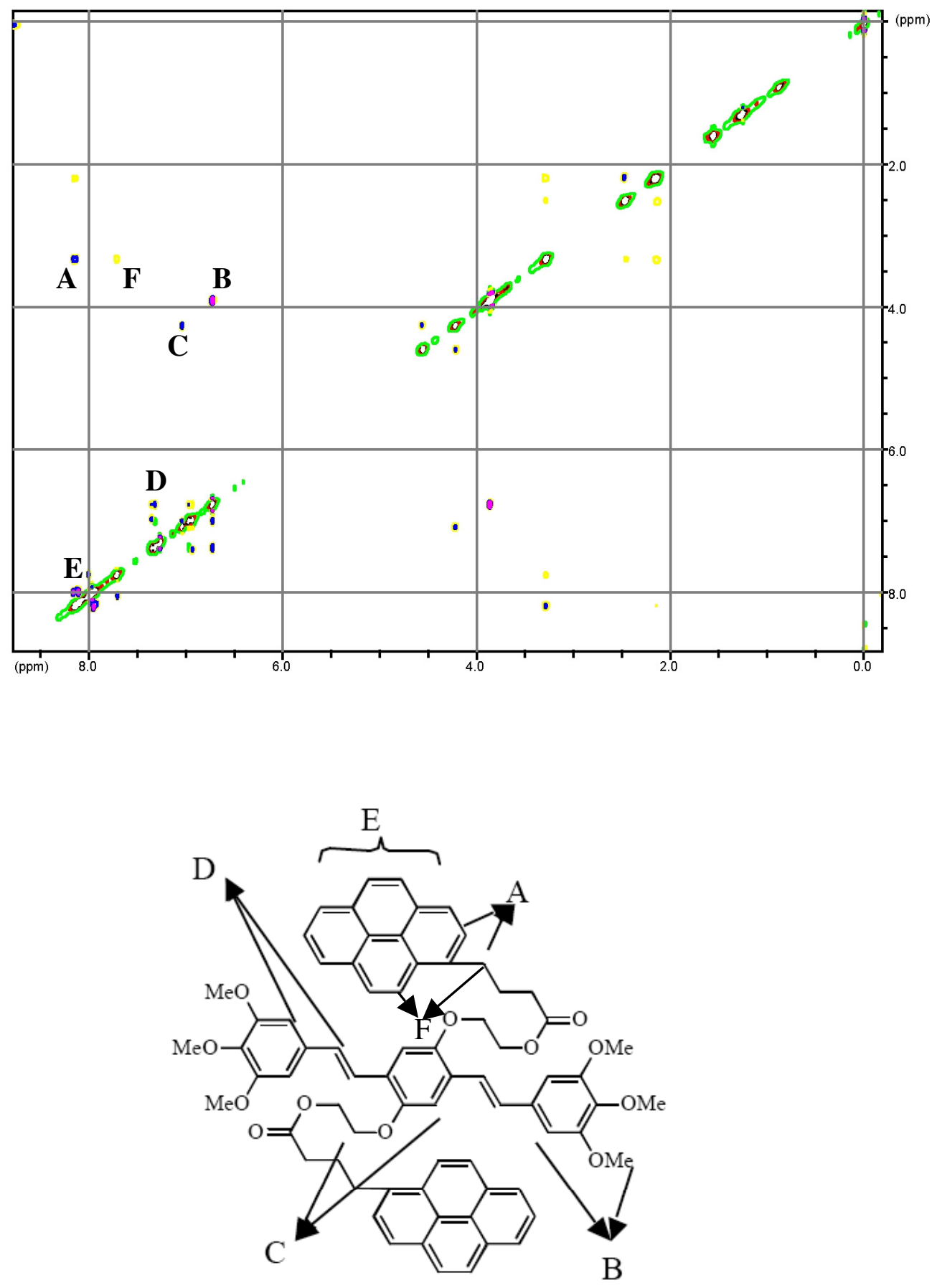


\section{Synthetic Details.}

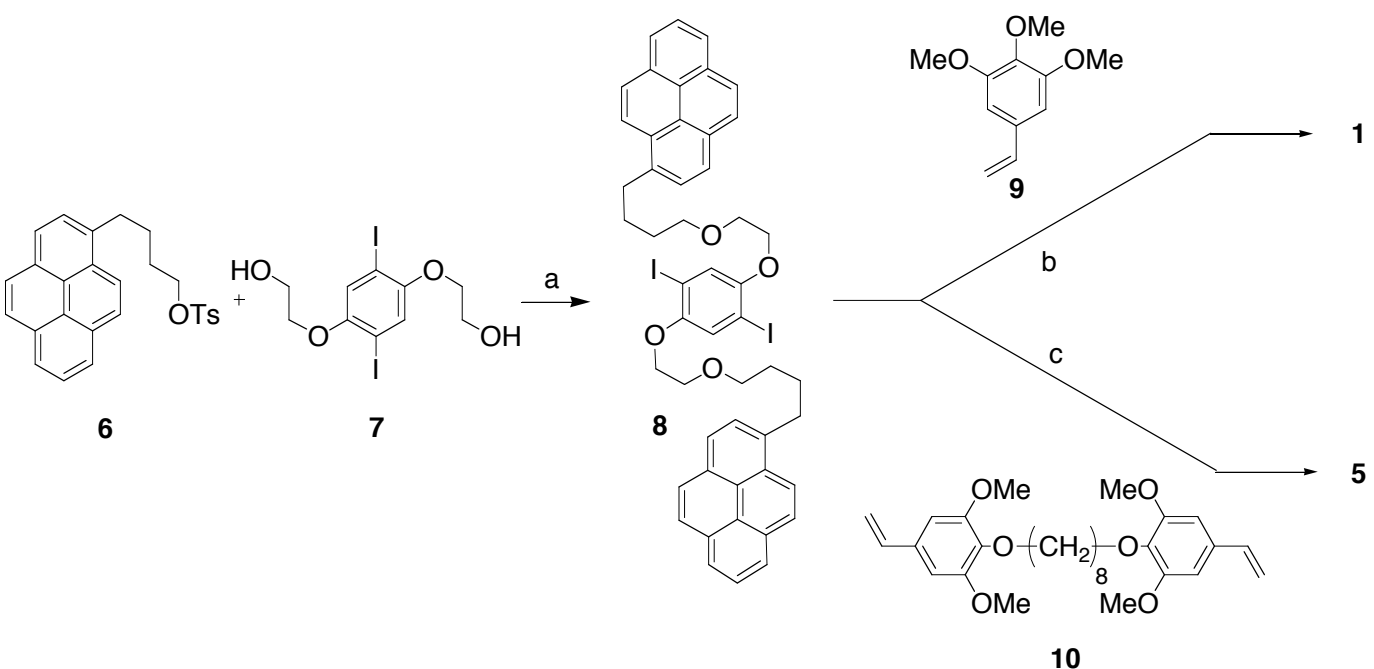<smiles>COc1cc(/C=C/c2cc(OCCO)c(/C=C/c3cc(OC)c(OC)c(OC)c3)cc2OCCO)cc(OC)c1OC</smiles>
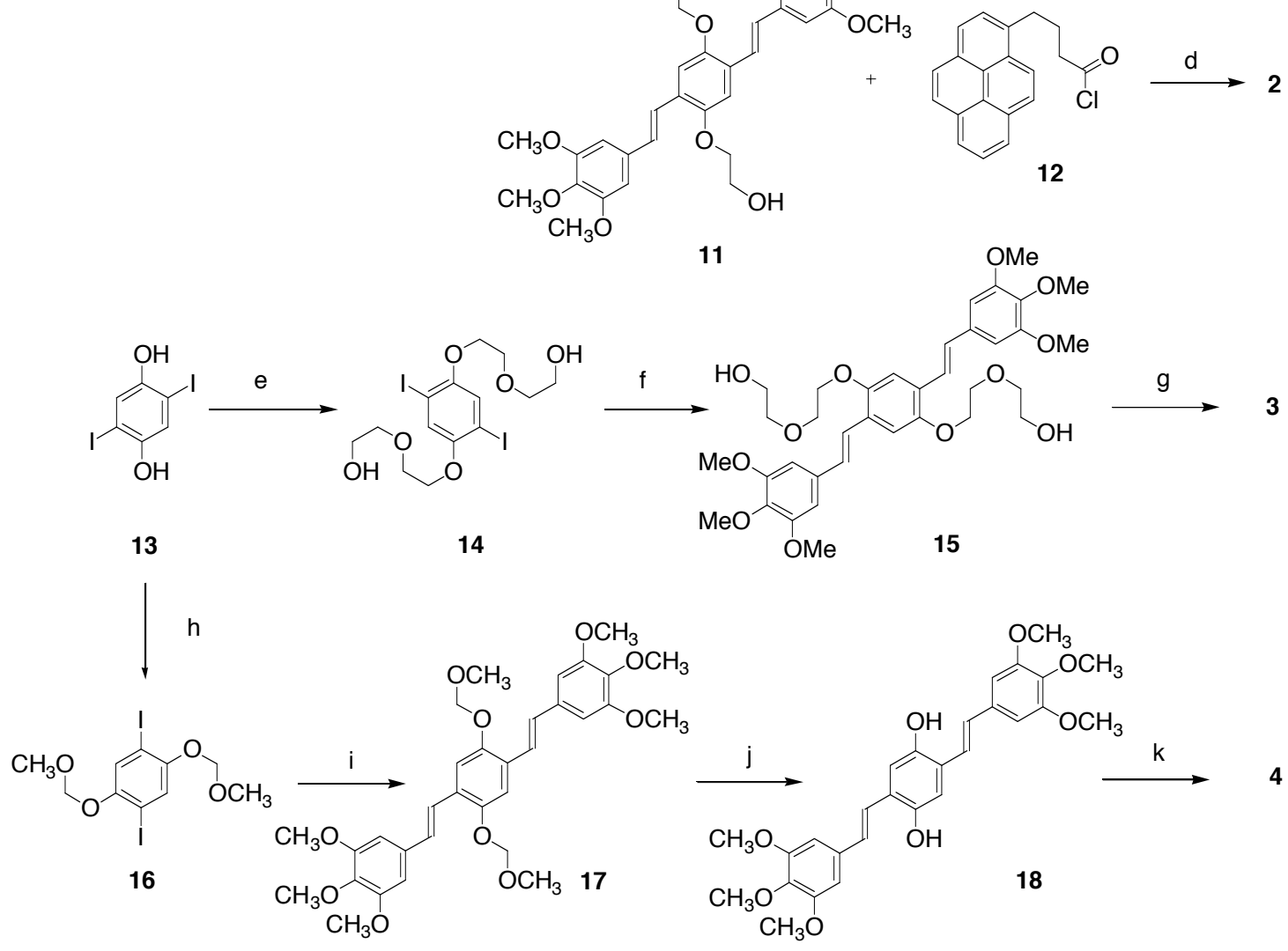

Scheme 1. Multichromophore syntheses. (a) NaH, THF, heat 7 days, $28 \%$. (b) $\mathrm{Pd}(\mathrm{OAc})_{2}, \mathrm{P}(o \text {-tolyl })_{3}, \mathrm{DMF} / \mathrm{NBu}_{3}, 85-90{ }^{\circ} \mathrm{C}, 76 \%$. (c) $\mathrm{Pd}(\mathrm{OAc})_{2}, \mathrm{P}(o \text {-tolyl })_{3}, \mathrm{DMF} / \mathrm{NBu}_{3}, 120-125^{\circ} \mathrm{C}, 40 \%$. (d) $\mathrm{NEt}_{3}, 24 \mathrm{~h}, 70 \%$. (e) 2-butanone, $\mathrm{K}_{2} \mathrm{CO}_{3}, 18$-crown-6, heat 7 days, $61 \%$. (f) $\mathrm{Pd}(\mathrm{OAc})_{2}, \mathrm{P}(o \text {-tolyl })_{3}, \mathrm{DMF} / \mathrm{NBu}_{3}, 120{ }^{\circ} \mathrm{C}, 77 \%$. (g) 12, $\mathrm{CH}_{2} \mathrm{Cl}_{2}, \mathrm{NEt}_{3} / \mathrm{DMAP}, 40 \%$. (h) $\mathrm{ClCH}_{2} \mathrm{OCH}_{3}, \mathrm{HN}(i-$

Pr $)_{2} / \mathrm{CH}_{2} \mathrm{Cl}_{2}, 94 \%$. (i) $\mathrm{Pd}(\mathrm{OAc})_{2}, \mathrm{P}(o \text {-tolyl })_{3}, \mathrm{DMF} / \mathrm{NBu}_{3}, 105^{\circ} \mathrm{C}, 91 \%$. (j) $p$-toluenesulfonic acid, r.t., $\mathrm{EtOH} / \mathrm{CH}_{2} \mathrm{Cl}_{2}, 84 \%$. (k) 4 (1-pyreny))butyric acid, $\mathrm{PPh}_{3} / \mathrm{NEt}_{3} / \mathrm{CCl}_{4}, \mathrm{CH}_{3} \mathrm{CN}, 48 \mathrm{~h}, 56 \%$. 
General Methods. All commercially available materials were used as received. Ether and THF were dried first over calcium hydride and then at reflux over sodium/benzophenone. Melting points are uncorrected. Molecular weights of polymers were measured by gel permeation chromatography in THF and referenced against linear polystyrene standards using a three-column system (Polymer Laboratories $300 \times 7.5 \mathrm{~mm}, 2$ mixed-D, $50 \AA$ ), a Knauer K-501 pump with a K-2301 refractive index detector and a K$2600 \mathrm{UV}$ detector $(395 \mathrm{~nm}) .{ }^{1} \mathrm{H}$ NMR and ${ }^{13} \mathrm{C}-\mathrm{NMR}$ spectral peak positions are reported relative to the internal reference specified in each case. Mass spectrometry was obtained under high-resolution fast atom bombardment or electron impact ionization conditions at the University of Massachusetts at Amherst Mass Spectrometry and Molecular Weight Analysis Facility. Relative quantum yields $\left(\phi_{\mathrm{FL}}\right)$ were measured using quinine sulfate in $0.10 \mathrm{M} \mathrm{H}_{2} \mathrm{SO}_{4}$ as a standard $\left(\phi_{\mathrm{FL}}=0.546\right)$, according to the procedure of Chen et al. [Chen, Z.; Huang, W.; Wang, L.; Kang, E.; Chen, B.; Lee, C. S.; Lee, S. T. Macromolecules 2000, 33, 9015].

4-(1-Pyrenyl)butyl tosylate (6). This compound was made by a literature procedure: $\mathrm{mp}$ 95-97 ${ }^{\circ} \mathrm{C}$ (lit mp 91-92 ${ }^{\circ} \mathrm{C}\left[\right.$ Winnik, F.; Winnik, M.; Tazuke, S.; Ober, C. Macromolecules 1987, 20, 38]). ${ }^{1} \mathrm{H}-$ NMR $\left(\mathrm{CDCl}_{3}\right): \delta 1.83(\mathrm{~m}, 4 \mathrm{H}), 2.34(\mathrm{~s}, 3 \mathrm{H}), 3.28(\mathrm{t}, 2 \mathrm{H}, \mathrm{J}=6.5 \mathrm{~Hz}), 4.11(\mathrm{t}, 2 \mathrm{H}, \mathrm{J}=6.5 \mathrm{~Hz}), 7.24,7.80$ (d, $4 \mathrm{H}, \mathrm{J}=8 \mathrm{~Hz}), 8.0-8.22(\mathrm{~m}, 9 \mathrm{H})$.

2,5-Diiodo-1,4-hydroquinol (13). This compound was made by a literature procedure: $\mathrm{mp}$ 197$199{ }^{\circ} \mathrm{C}$ (lit. mp 195-197 ${ }^{\circ} \mathrm{C}$ [Winnik, F.; Winnik, M.; Tazuke, S.; Ober, C. Macromolecules 1987, 20, 38]). ${ }^{1} \mathrm{H}-\mathrm{NMR}$ (acetone- $d_{6}$ ): $\delta 7.29$ (s, $\left.2 \mathrm{H}\right), 8.82(\mathrm{~s}, 2 \mathrm{H}) ;{ }^{13} \mathrm{C}-\mathrm{NMR}$ (acetone $\left.-d_{6}\right)$ : $\delta 83.15,123.65,150.43$.

1,4-Bis(2-hydroxyethoxy)-2,5-diiodobenzene (7). This compound was made from 13 by a previously published procedure[Sierra, C. A.; Lahti, P. M. Chem. Mater. 2004, 16, 55]: mp 169-170 ${ }^{\circ} \mathrm{C}$. ${ }^{1} \mathrm{H}-\mathrm{NMR}$ (DMSO- $\left.d_{6}\right): \delta 3.67(\mathrm{~m}, 4 \mathrm{H}), 3.97(\mathrm{t}, 4 \mathrm{H}, \mathrm{J}=5 \mathrm{~Hz}), 4.83(\mathrm{t}, 2 \mathrm{H}, \mathrm{J}=5 \mathrm{~Hz}), 7.36(\mathrm{~s}, 2 \mathrm{H}) ;{ }^{13} \mathrm{C}-$ NMR (DMSO- $\left.d_{6}\right): 60.55,72.80,88.00,123.99,153.60$.

2,5-Bis(2-[4-(1-pyrenyl)butoxy]ethoxy)-1,4-diiodobenzene (8). Under an atmosphere of argon at room temperature, $7(549 \mathrm{mg}, 1.22 \mathrm{mmol})$ was added to a suspension of $95 \%$ dry sodium hydride (381 $\mathrm{mg}, 15.8 \mathrm{mmol})$ in $20 \mathrm{~mL}$ of dry THF. After $30 \mathrm{~min}$ of stirring, $6(1.15 \mathrm{~g}, 2.68 \mathrm{mmol})$ in $10 \mathrm{~mL}$ of dry THF was added dropwise. The mixture was stirred for 7 days at $75{ }^{\circ} \mathrm{C}$ and then quenched by addition of a few drops of water (caution! exothermic reaction, potential fire hazard). After concentration under vacuum, the residue was twice partitioned between dichloromethane and water. The combined organic phases were dried over anhydrous $\mathrm{MgSO}_{4}$, filtered, evaporated and purified by column chromatography (silica, dichloromethane) to yield $327 \mathrm{mg}$ (28\%) of a yellowish solid with mp 134-136 ${ }^{\circ} \mathrm{C}$. HRMS (FAB, $\mathrm{m} / \mathrm{z}$ ): calcd for $\mathrm{C}_{50} \mathrm{H}_{44} \mathrm{I}_{2} \mathrm{O}_{4} 962.1329$, found 962.1498. ${ }^{1} \mathrm{H}-\mathrm{NMR}\left(\mathrm{CDCl}_{3}\right): \delta 1.82(\mathrm{~m}, 4 \mathrm{H}), 1.96(\mathrm{~m}, 4 \mathrm{H})$, $3.36(\mathrm{t}, 4 \mathrm{H}, \mathrm{J}=8 \mathrm{~Hz}), 3.62(\mathrm{t}, 4 \mathrm{H}, \mathrm{J}=6 \mathrm{~Hz}), 3.76(\mathrm{t}, 4 \mathrm{H}, \mathrm{J}=5 \mathrm{~Hz}), 4.04(\mathrm{t}, 4 \mathrm{H}, \mathrm{J}=5 \mathrm{~Hz}), 7.19(\mathrm{~s}, 2 \mathrm{H})$, 7.87-8.29 (m, $18 \mathrm{H}) .{ }^{13} \mathrm{C}-\mathrm{NMR}\left(400 \mathrm{MHz}, \mathrm{CDCl}_{3}\right): 28.39,29.81,33.30,69.19,70.31,71.59,86.48$, $123.51,124.65,124.78,125.08,125.75,126.52,127.16,127.30,127.53,128.65,129.76,130.93,131.44$, $137.10,153.15$.

3,4,5-Trimethoxystyrene (9). This compound was made by a literature procedure as a yellowish oil [Gangjee, A.; Devraj, R.; Queener, S. F. J. Med. Chem. 1997, 40, 470]. ${ }^{1} \mathrm{H}-\mathrm{NMR}\left(\mathrm{CDCl}_{3}\right)$ : $\delta 3.81$ (s, 3 $\mathrm{H}), 3.82(\mathrm{~s}, 6 \mathrm{H}), 5.17\left(\mathrm{dd}, 1 \mathrm{H}, \mathrm{J}=10.8 \mathrm{~Hz}, \mathrm{~J}{ }^{\prime}=0.6\right), 5.62(\mathrm{dd}, 1 \mathrm{H}, \mathrm{J}=18 \mathrm{~Hz}, \mathrm{~J}$ '= 0.6), 6.58 (dd, $1 \mathrm{H}, \mathrm{J}=$ $10.8 \mathrm{~Hz}, \mathrm{~J} '=10.8 \mathrm{~Hz}), 6.59(\mathrm{~s}, 2 \mathrm{H})$.

E,E-2,5-Bis(2-[4-(1-pyrenyl)butoxy]ethoxy)-1,4-bis((3,4,5-trimethoxystyryl)-benzene (1). A $50 \mathrm{~mL}$ round-bottom flask was fitted with a condenser, charged with $8(103 \mathrm{mg}, 0.107 \mathrm{mmol}), \mathbf{9}(41 \mathrm{mg}$, $0.214 \mathrm{mmol})$, tri $(o$-tolyl $)$ phosphine $(5.5 \mathrm{mg}, 0.02 \mathrm{mmol})$ and $\mathrm{Pd}(\mathrm{OAc})_{2}(1 \mathrm{mg}, 0.004 \mathrm{mmol})$, then flushed with argon. Next $10 \mathrm{~mL}$ of dry DMF was added, the solution was heated to $85-90{ }^{\circ} \mathrm{C}$, and $0.05 \mathrm{~mL}$ of triethylamine added. After $24 \mathrm{~h}$ of stirring with heat, the reaction mixture was cooled to room temperature, poured into $100 \mathrm{~mL}$ of water, and extracted twice with ether. The solvent was evaporated and the residue subjected to column chromatography (silica, 94:6 chloroform: acetone). The isolated product fraction was evaporated under vacuum and washed with diethyl ether to give bright yellow product $\left(90 \mathrm{mg}, 76 \%, \mathrm{mp} 65-71{ }^{\circ} \mathrm{C}\right)$. $\mathrm{HRMS}\left(\mathrm{FAB}, \mathrm{m} / \mathrm{z}\right.$ ): calcd for $\mathrm{C}_{72} \mathrm{H}_{70} \mathrm{O}_{10} 1094.4969$, found 1094.5305. UV-vis (chloroform, $\lambda_{\max } / \mathrm{nm}[\varepsilon]$ ]): 245[50700], 269[22600], 276[36300], 330[27900], 
346[37500], 387 broad[10300]. ${ }^{1} \mathrm{H}-\mathrm{NMR}\left(\mathrm{CDCl}_{3}\right): \delta 1.83(\mathrm{~m}, 4 \mathrm{H}), 1.94(\mathrm{~m}, 4 \mathrm{H}), 3.32$ (t, $\left.4 \mathrm{H}, \mathrm{J}=8 \mathrm{~Hz}\right)$, $3.64(\mathrm{t}, 4 \mathrm{H}, \mathrm{J}=6 \mathrm{~Hz}$ ), 3.83 (overlapping s plus t, $22 \mathrm{H}), 4.22(\mathrm{t}, 4 \mathrm{H}, \mathrm{J}=5 \mathrm{~Hz}), 6.68(\mathrm{~s}, 4 \mathrm{H}), 7.01$ (d, $2 \mathrm{H}$, $\mathrm{J}=16 \mathrm{~Hz}), 7.34(\mathrm{~d}, 2 \mathrm{H}, \mathrm{J}=16 \mathrm{~Hz}), 7.14(\mathrm{~s}, 2 \mathrm{H}), 7.77(\mathrm{~d}, 2 \mathrm{H}, \mathrm{J}=8 \mathrm{~Hz}), 7.90-8.24(\mathrm{~m}, 18 \mathrm{H}) .{ }^{13} \mathrm{C}-\mathrm{NMR}$ (400 MHz, $\mathrm{CDCl}_{3}$ ): 28.33, 29.69, 33.16, 56.04, 60.95, 69.33, 69.53, 71.44, 103.67, 122.82, 123.35, $124.64,124.74,124.78,124.98,125.74,126.52,127.17,127.48,129.10,130.86,131.86,136.62,153.30$.

1,8-Bis(2,6-dimethoxy-4-vinylphenoxy)-octane (10). This compound was made by a literature procedure: $\mathrm{mp} 52-54{ }^{\circ} \mathrm{C}$ (lit. mp 52-53 ${ }^{\circ} \mathrm{C}$ Pasco, S.; Lahti, P. M.; Karasz, F. E. Macromolecules 1999, 32, 6933]). ${ }^{1} \mathrm{H}-\mathrm{NMR}\left(\mathrm{CDCl}_{3}\right): \delta$ 1.34-1.46 (m, $\left.8 \mathrm{H}\right), 1.76(\mathrm{~m}, 4 \mathrm{H}), 3.85(\mathrm{~m}, 12 \mathrm{H}), 3.95(\mathrm{t}, 4 \mathrm{H}, \mathrm{J}=7 \mathrm{~Hz})$, $5.20(\mathrm{~d}, 2 \mathrm{H}, \mathrm{J}=12 \mathrm{~Hz}), 5.65(\mathrm{~d}, 2 \mathrm{H}, \mathrm{J}=18 \mathrm{~Hz}), 6.62(\mathrm{~s}, 4 \mathrm{H}), 6.63(\mathrm{~d}, 2 \mathrm{H}, \mathrm{J}=11 \mathrm{~Hz})$.

Polymer (5). Compounds 8 (100 mg, $0.1 \mathrm{mmol})$ and $\mathbf{1 0}(49 \mathrm{mg}, 0.1 \mathrm{mmol})$, tri (o-tolyl)phosphine $(6 \mathrm{mg}, 0.02 \mathrm{mmol})$ and $\mathrm{Pd}(\mathrm{OAc})_{2}(0.9 \mathrm{mg}, 4$ micromoles$)$ were placed in a $50 \mathrm{~mL}$ round-bottom flask fitted with a condenser and flushed with argon. Then, $10 \mathrm{~mL}$ of dry DMF and $0.1 \mathrm{~mL}$ of tributylamine were added, and the mixture heated to $120-125^{\circ} \mathrm{C}$. After $72 \mathrm{~h}$ the reaction mixture was cooled to room temperature, and then poured into $100 \mathrm{~mL}$ of ice water. The resulting yellow, paste-like product covering the flask wall was dissolved in dichloromethane, concentrated with a stream of air, and dried under vacuum overnight to yield $50 \mathrm{mg}(40 \%)$ of 5 . UV-vis (THF, $\left.\lambda_{\max } / \mathrm{nm}[\varepsilon]\right): 242[67000], 264[35500]$, 276[48000], 315[22600], 328[40500], 344[51300], 375 broad[10000]. GPC (THF): $\mathrm{M}_{\mathrm{n}}=9100, \mathrm{M}_{\mathrm{w}}=$ 15700. ${ }^{1} \mathrm{H}-\mathrm{NMR}\left(\mathrm{CDCl}_{3}\right): \delta 1.27-143(\mathrm{~m}, 10 \mathrm{H}), 1.75-1.92(\mathrm{~m}, 14 \mathrm{H}), 3.28(\mathrm{~m}, 6 \mathrm{H}), 3.62(\mathrm{~m}, 4 \mathrm{H}), 3.85$ (m, 12 H), $3.94(\mathrm{~m}, 6 \mathrm{H}), 4.20$ (m, $4 \mathrm{H}), 6.62(\mathrm{~m}, 2 \mathrm{H}), 6.70$ (m, $2 \mathrm{H}), 7.03(\mathrm{~m}, 2 \mathrm{H}), 7.14(\mathrm{~s}, 1 \mathrm{H}), 7.30$ $(\mathrm{m}, 2 \mathrm{H}), 7.79-8.26(\mathrm{~m}, 20 \mathrm{H})$. A small residuum of vinyl end group resonances was observed at $\delta$ 5.2-5.5 in the ${ }^{1} \mathrm{H}-\mathrm{NMR} .{ }^{13} \mathrm{C}-\mathrm{NMR}\left(\mathrm{CDCl}_{3}\right)$ : 22,68, 25.30, 25.84, 28.33, 29.46, 29.76, 30.10, 31.61, 33.18, 34.68, $56.11,69.32,71.44,73.59,103.39,103.83,123.38,124.79,125.00,125.76,126.54,127.19,127.51$, $128.58,129.74,130.88,131.40,133.03,133.34,136.85,137.30,151.09,153.56$.

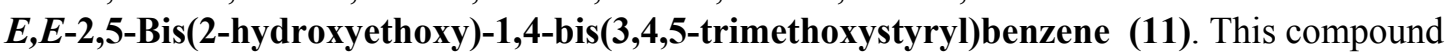
was made from 7 and 9 by a previously published procedure[Sierra, C. A.; Lahti, P. M. Chem. Mater. 2004, 16, 55]: $\mathrm{mp} 245-247{ }^{\circ} \mathrm{C} .{ }^{1} \mathrm{H}-\mathrm{NMR}$ (DMSO- $d_{6}$ ): $\delta 3.67$ (s, $\left.6 \mathrm{H}\right), 3.82$ (overlapping s plus t, $16 \mathrm{H}$ ), 4.08 (t, 4 H, J = 4 Hz), 6.89 (s, 4 H), 7.3 (d, 2 H, J=16.8 Hz), 7.45 (d, 2 H, J= 16.8 Hz), 7.34 (s, 2 H).

4-(1-Pyrenyl)butyryl chloride (12). This compound was made by literature procedures as a pale yellow solid with $\mathrm{mp} 81-83^{\circ} \mathrm{C}$ (lit mp 73.5-74.5 ${ }^{\circ} \mathrm{C}$ Tran, C. D.; Fendler, J. H. J. Am. Chem. Soc. 1980, 102, 2923], lit mp 89-90 ${ }^{\circ} \mathrm{C}$ [Winnik, M. A.; Redpath, T.; Richards, D. H. Macromolecules 1980, 13 , 328]). ${ }^{1} \mathrm{H}-\mathrm{NMR}$ (DMSO- $d_{6}$ ): $\delta 2.01(\mathrm{~m}, 2 \mathrm{H}), 2.46$ (t, $\left.2 \mathrm{H}, \mathrm{J}=7 \mathrm{~Hz}\right), 3.32$ (t, $\left.2 \mathrm{H}, \mathrm{J}=8 \mathrm{~Hz}\right), 7.90$ (d, $2 \mathrm{H}$ ). IR (NaCl plate, $\mathrm{cm}^{-1}$ ): 3040, 2949, 2875, 1732, 1587, 1435, 1372, 1145, 1036, 843, 758, 720, 681; no OH stretch was observed in $3500-2500 \mathrm{~cm}^{-1}$ region.

$E, E-2,5-B i s(2-[4-(1-p y r e n y l) b u t y r y l o x y]$ ethoxy)-1,4-bis[2-(3,4,5-trimethoxystyryl)-benzene (2). In a round bottom flask at $0{ }^{\circ} \mathrm{C}$ under argon were placed $11(122 \mathrm{mg}, 0.21 \mathrm{mmol})$ and triethylamine $(0.2 \mathrm{~mL}, 1.05 \mathrm{mmol})$. After stirring for $30 \mathrm{~min}, 12(135 \mathrm{mg}, 0.44 \mathrm{mmol})$ was added. The reaction mixture was warmed to room temperature and allowed to stir for $24 \mathrm{~h}$. Addition of hexanes to the resulting mixture precipitated a fibrous yellow solid. Filtration and precipitation from chloroform:hexanes yielded $165 \mathrm{mg}(70 \%)$ of greenish-yellow powder with mp 162-164 ${ }^{\circ} \mathrm{C}$. HRMS(FAB, m/z): calcd for $\mathrm{C}_{72} \mathrm{H}_{66} \mathrm{O}_{12}$ 1122.4554, found 1122.4496. ${ }^{1} \mathrm{H}-\mathrm{NMR}\left(\mathrm{CDCl}_{3}\right): \delta 2.15(\mathrm{~m}, 4 \mathrm{H}), 2.46(\mathrm{t}, 4 \mathrm{H}, \mathrm{J}=7 \mathrm{~Hz}), 3.28(\mathrm{t}, 4 \mathrm{H}, \mathrm{J}=7$ $\mathrm{Hz}), 3.84(\mathrm{~s}, 4 \mathrm{H}), 3.86(\mathrm{~s}, 14 \mathrm{H}), 4.22(\mathrm{t}, 4 \mathrm{H}, \mathrm{J}=5 \mathrm{~Hz}), 4.55(\mathrm{t}, 4 \mathrm{H}, \mathrm{J}=5 \mathrm{~Hz}), 6.72(\mathrm{~s}, 4 \mathrm{H}), 6.95(\mathrm{~d}, 2 \mathrm{H}$, $\mathrm{J}=16 \mathrm{~Hz}), 7.04(\mathrm{~s}, 2 \mathrm{H}), 7.33(\mathrm{~d}, 2 \mathrm{H}, \mathrm{J}=16 \mathrm{~Hz}), 7.72(\mathrm{~d}, 2 \mathrm{H}, \mathrm{J}=8 \mathrm{~Hz}), 7.92(\mathrm{~m}, 10 \mathrm{H}), 8.12(\mathrm{~m}, 6 \mathrm{H})$. ${ }^{13} \mathrm{C}-\mathrm{NMR}\left(\mathrm{CDCl}_{3}\right): 26.92,32.84,34.20,56.43,61.27,67.95,104.02,121.95,122.59,123.45,125.05$, $125.18,125.28,126.10,126.97,127.23,127.47,127.61,127.74,128.95,129.69,130.20,131.13,131.66$, 133.70, 135.72, 138.30, 151.09, 153.68, 173.64. FTIR $\left(\mathrm{KBr}\right.$ pellet, $\left.\mathrm{cm}^{-1}\right): 3035,2934,1735,1580,1507$, $1419,1322,1244,1127,1005,962,844$. UV-vis (THF, $\left.\lambda_{\max } / \mathrm{nm}[\varepsilon]\right): 243[94600], 264[47200]$, 276[70100], 327[75000], 343[97000], 397[48200].

2,5-Diiodo-1,4-bis[2-(2-hydroxyethoxy)ethoxy]benzene. (14). This compound was made by a literature procedure as a pale yellow solid with $\mathrm{mp} 104-106{ }^{\circ} \mathrm{C}$ (lit mp 104-105.5 ${ }^{\circ} \mathrm{C}[\mathrm{Zhou}, \mathrm{Q}$.; Swager, 
T. J. Am. Chem. Soc. 1995, 117, 12593]). ${ }^{1} \mathrm{H}-\mathrm{NMR}\left(\mathrm{CDCl}_{3}\right): \delta 2.15$ (br s, $\left.2 \mathrm{H}\right), 3.70$ (t, $\left.4 \mathrm{H}, \mathrm{J}=4.8 \mathrm{~Hz}\right)$, 3.76 (s, $4 \mathrm{H}), 3.88$ (t, $4 \mathrm{H}, \mathrm{J}=4.5 \mathrm{~Hz}), 4.12$ (t, $4 \mathrm{H}, \mathrm{J}=4.7 \mathrm{~Hz}), 7.23$ (s, $2 \mathrm{H})$.

E,E-2,5-Bis(2-(2-hydroxyethoxy)ethoxy)-1,4-bis(3,4,5-trimethoxystyryl)benzene (15).

Compounds 9 (0.64 g, $3.3 \mathrm{mmol})$ and $14(0.71 \mathrm{~g}, 1.32 \mathrm{mmol})$, tri (o-tolyl)phosphine (200 mg, $0.66 \mathrm{mmol})$ and $\mathrm{Pd}(\mathrm{OAc})_{2}(30 \mathrm{mg}, 0.13 \mathrm{mmol})$ were placed in a $50 \mathrm{~mL}$ round-bottom flask fitted with a condenser were flushed with argon, and $20 \mathrm{~mL}$ of dry DMF was added. The reaction was heated to $120^{\circ} \mathrm{C}$, and 1.2 $\mathrm{mL}$ of triethylamine added. After 2 days of heating, the reaction was cooled to room temperature and poured into $200 \mathrm{~mL}$ of water to give a dark brown precipitate that was dissolved in dichloromethane, filtered through Celite, concentrated and precipitated from dichloromethane:hexanes to yield $680 \mathrm{mg}$ (77\%) of yellow powder product with mp $225-228^{\circ} \mathrm{C}$. HRMS(ESI, m/z): calcd for $\mathrm{C}_{36} \mathrm{H}_{46} \mathrm{O}_{12}\left(\mathrm{M}^{+}+\mathrm{H}\right)$ 670.2996, found 670.2989. ${ }^{1} \mathrm{H}-\mathrm{NMR}\left(\mathrm{CDCl}_{3}\right)$ : $\delta 3.70(\mathrm{t}, 4 \mathrm{H}, \mathrm{J}=4.4 \mathrm{~Hz}), 3.76(\mathrm{~m}, 4 \mathrm{H}), 3.87(\mathrm{~s}, 6 \mathrm{H})$, 3.92 (overlapping s plus t, $14 \mathrm{H}), 4.25(\mathrm{t}, 4 \mathrm{H}, \mathrm{J}=4.6 \mathrm{~Hz}), 6.77(\mathrm{~s}, 4 \mathrm{H}), 7.06(\mathrm{~d}, 2 \mathrm{H}, \mathrm{J}=16 \mathrm{~Hz}), 7.34(\mathrm{~d}, 2$ $\mathrm{H}, \mathrm{J}=16 \mathrm{~Hz}), 7.15(\mathrm{~s}, 2 \mathrm{H}) ;{ }^{13} \mathrm{C}-\mathrm{NMR}\left(\mathrm{CDCl}_{3}\right): 56.71,61.00,61.76,69.24,69.90,72.57,103.80,111.40$, $122.87,127.70,129.41,133.40,138.02,151.02,153.42$.

\section{E,E-2,5-Bis(2-[2-(4-[1-pyrenyl]butyryloxy)-ethoxy]-ethoxy)-1,4-bis(3,4,5-}

trimethoxystyryl)benzene (3). A round bottom flask containing 4-(1-pyrene)butyric acid (150 $\mathrm{mg}, 0.52$ $\mathrm{mmol}$ ) in $20 \mathrm{~mL}$ of fresh dry dichloromethane and $0.25 \mathrm{~mL}$ of dry DMF under argon was cooled to $0{ }^{\circ} \mathrm{C}$. Then thionyl chloride $(0.2 \mathrm{~mL}, 2.7 \mathrm{mmol})$ was added and the reaction was stirred for $1 \mathrm{~h}$ at room temperature to give a clear solution, which was then heated to reflux. After $12 \mathrm{~h}$ the reaction was complete according to TLC analysis. Excess thionyl chloride and solvent were then removed by distillation under reduced pressure, and the resulting solid product dissolved in a minimal amount of dichloromethane to make a solution of $\mathbf{1 2}$.

In a round bottom flask under argon $15(70 \mathrm{mg}, 0.1 \mathrm{mmol})$ was added to $15 \mathrm{~mL}$ of dry dichloromethane. The suspension was stirred and cooled to $0{ }^{\circ} \mathrm{C}$ in an ice bath. Triethylamine $(30 \mathrm{microL}$, $0.2 \mathrm{mmol}$ ) and 4-(dimethylamino)pyridine (DMAP, $64 \mathrm{mg}, 0.52 \mathrm{mmol}$ ) were added. After $30 \mathrm{~min}$ the solution of 12 in dichloromethane was added and the reaction was allowed to stir at room temperature for $24 \mathrm{~h}$. The reaction was then poured into $100 \mathrm{~mL}$ of water, and the organic layer separated, dried over $\mathrm{MgSO}_{4}$, filtered and purified by column chromatography over silica gel (dichloromethane:methanol 95:5) to give $50 \mathrm{mg}(40 \%)$ of product with $\mathrm{mp} 107-110{ }^{\circ} \mathrm{C}$. $\mathrm{HRMS}(\mathrm{FAB}, \mathrm{m} / \mathrm{z})$ calcd for $\mathrm{C}_{76} \mathrm{H}_{74} \mathrm{O}_{14} 1210.5247$, found 1210.5079. ${ }^{1} \mathrm{H}-\mathrm{NMR}\left(\mathrm{CDCl}_{3}\right): \delta 2.15(\mathrm{~m}, 4 \mathrm{H}), 2.45(\mathrm{t}, 4 \mathrm{H}, \mathrm{J}=8 \mathrm{~Hz}), 3.33(\mathrm{t}, 4 \mathrm{H}, \mathrm{J}=8 \mathrm{~Hz}), 3.81$ (t, $4 \mathrm{H}, \mathrm{J}=4 \mathrm{~Hz}$ ), 3.89 (overlapping s plus t, $20 \mathrm{H}$ ), 4.17 (t, $4 \mathrm{H}, \mathrm{J}=8 \mathrm{~Hz}), 4.31$ (t, $4 \mathrm{H}, \mathrm{J}=4 \mathrm{~Hz}$ ), 6.73 (s, 4 H), 6.99 (d, 2 H, J=16 Hz), 7.29 (d, 2 H, J=16 Hz), 7.06 (s, 2 H), 7.80 (d, 2 H, J=8 Hz), 7.97 (m, $10 \mathrm{H})$, $8.12(\mathrm{~m}, 8 \mathrm{H}) ;{ }^{13} \mathrm{C}-\mathrm{NMR}\left(\mathrm{CDCl}_{3}\right): 26.70,30.95,32.20,33.75,56.12,61.27,63.35,69.05,69.45,69.95$, $103.69,122.59,123.35,124.75,124.87,124.98,125.10,125.97,126.63,127.07,127.34,127.46,128.68$, $129.69,129.90,130.13,131.36,133.50,135.72,137.30,150.92,153.38,173.41$. UV-vis (THF, $\lambda_{\max } / \mathrm{nm}$ [ع]): 243[103800], 266[41300], 277[64500], 328[61000], 344[84550], 375[32300].

1,4-Diiodo-2,5-bis(methoxymethoxy)benzene (16). A round bottom flask containing 13 (514 $\mathrm{mg}, 1.42 \mathrm{mmol})$ in $20 \mathrm{~mL}$ of dry dichloromethane was cooled to $0{ }^{\circ} \mathrm{C}$. Then, $1 \mathrm{~mL}$ of $N, N$ -

diisopropylethylamine $(5.7 \mathrm{mmol})$ was added dropwise. The reaction was allowed to stir for $30 \mathrm{~min}$, and then chloromethyl methyl ether $(0.32 \mathrm{~mL}, 4.26 \mathrm{mmol})$ was added, turning the reaction mixture from a suspension to a clear solution after a few minutes. After $4 \mathrm{~h}$ of stirring at room temperature, $20 \mathrm{~mL}$ of water was added. The organic layer was separated, washed three times with water, dried over $\mathrm{MgSO}_{4}$, and vacuum concentrated. Several precipitations from dichloromethane:hexanes gave $600 \mathrm{mg}(94 \%)$ of product as light brown needles with mp $128-130{ }^{\circ} \mathrm{C}$. MS(ESI, m/z): calcd for $\mathrm{C}_{10} \mathrm{H}_{12} \mathrm{O}_{4} \mathrm{I}_{2}\left(\mathrm{M}^{+}+\mathrm{H}\right)$ 449.8824, found 449.8436. ${ }^{1} \mathrm{H}-\mathrm{NMR}\left(\mathrm{CDCl}_{3}\right)$ : $\delta 3.51(\mathrm{~s}, 6 \mathrm{H}), 5.15(\mathrm{~s}, 4 \mathrm{H}), 7.45(\mathrm{~s}, 2 \mathrm{H})$.

$\boldsymbol{E}, \boldsymbol{E}$-2,5-Bis(methoxymethoxy)-1,4-bis(3,4,5-trimethoxystyryl)benzene.(17). A $50 \mathrm{~mL}$ roundbottom flask was fitted with a condenser, charged with $16(580 \mathrm{mg}, 1.3 \mathrm{mmol}), \mathbf{9}(500 \mathrm{mg}, 2.6 \mathrm{mmol})$, tri(o-tolyl)phosphine (79 $\mathrm{mg}, 0.26 \mathrm{mmol})$ and $\mathrm{Pd}(\mathrm{OAc})_{2}(12 \mathrm{mg}, 0.05 \mathrm{mmol})$, then flushed with argon. Then $10 \mathrm{~mL}$ of dry DMF and $1 \mathrm{~mL}$ of triethylamine were added and the reaction mixture was warmed to $105{ }^{\circ} \mathrm{C}$, stirred for $48 \mathrm{~h}$, and then cooled to room temperature. The reaction was poured into $100 \mathrm{~mL}$ of 
water to give a yellow cloudy suspension that was extracted with dichloromethane. The organic layer was dried over $\mathrm{MgSO}_{4}$, vacuum concentrated and purified by column chromatography (silica, dichloromethane) to yield $688 \mathrm{mg}(91 \%)$ of a reddish powder with $\mathrm{mp} 214-216^{\circ} \mathrm{C}$. This material was used without further purification for the next synthetic step. ${ }^{1} \mathrm{H}-\mathrm{NMR}\left(\mathrm{CDCl}_{3}\right): \delta 3.57(\mathrm{~s}, 6 \mathrm{H}), 3.87(\mathrm{~s}, 7$ H), 3.93 (overlapping s, $13 \mathrm{H}), 5.27(\mathrm{~s}, 4 \mathrm{H}), 6.76(\mathrm{~s}, 4 \mathrm{H}), 7.04(\mathrm{~d}, 4 \mathrm{H}, \mathrm{J}=16 \mathrm{~Hz}), 7.33(\mathrm{~d}, 4 \mathrm{H}, \mathrm{J}=16$ $\mathrm{Hz}), 7.37$ (s, $2 \mathrm{H}) .{ }^{13} \mathrm{C}-\mathrm{NMR}\left(\mathrm{CDCl}_{3}\right): 56.35,56.44,61.00,95.75,103.14,112.83,122.43,127.60,129.70$, $133.42,138.00,150.09,153.39$.

$\boldsymbol{E}, \boldsymbol{E}$-1,4-Bis(3,4,5-trimethoxystyryl)-2,5-dihydroxybenzene (18). To a suspension of 17 (688 $\mathrm{mg}, 1,2 \mathrm{mmol})$ in $10 \mathrm{~mL}$ of ethanol was added $p$-toluenesulfonic acid monohydrate $(1 \mathrm{~g}, 5.5 \mathrm{mmol})$. The reaction was stirred for $1 \mathrm{~h}$ at room temperature, and then $8 \mathrm{~mL}$ of dichloromethane was added to give a clear solution. After $24 \mathrm{~h}$ the reaction was treated with $50 \mathrm{~mL}$ of $5 \%$ aq $\mathrm{HCl}$ and extracted with dichloromethane, dried over $\mathrm{MgSO}_{4}$, filtered and purified by column chromatography over silica gel (dichloromethane /methanol 95:5) to give $500 \mathrm{mg}$ (84\%) of a light brown powder with $\mathrm{mp} 220-224{ }^{\circ} \mathrm{C}$. MS(ESI, m/z): calcd for $\mathrm{C}_{28} \mathrm{H}_{30} \mathrm{O}_{8}\left(\mathrm{M}^{+}+\mathrm{H}\right) 494.19$, found 494.20. ${ }^{1} \mathrm{H}-\mathrm{NMR}$ (acetone- $\left.d_{6}\right)$ : $\delta 3.75(\mathrm{~s}, 6 \mathrm{H})$, 3.89 (s, $12 \mathrm{H}), 6.88$ (s, $4 \mathrm{H}), 7.11$ (d, $2 \mathrm{H}, \mathrm{J}=16 \mathrm{~Hz}), 7.44$ (d, $2 \mathrm{H}, \mathrm{J}=16 \mathrm{~Hz}) .7 .14$ (s, $2 \mathrm{H}) .{ }^{13} \mathrm{C}-\mathrm{NMR}$ (acetone- $d_{6}$ ): 61.00, 65.14, 109.83, 128.83, 129.90, 133.70, 139.42, 143.50, 153.09, 159.39.

E,E-2,5-Bis(4-(1-pyrenyl)butyryloxy)-1,4-bis(3,4,5-trimethoxystyryl)benzene (4). A mixture of 17 (153 mg, $0.31 \mathrm{mmol}$ ), pyrenebutyric acid (200 mg, $0.69 \mathrm{mmol})$, triphenylphospine (182 $\mathrm{mg}, 0.69$ $\mathrm{mmol})$, triethylamine $(0.4 \mathrm{~mL}, 2.87 \mathrm{mmol})$ and $4 \mathrm{~mL}$ of carbon tetrachloride in $15 \mathrm{~mL}$ of acetonitrile was stirred at room temperature for $48 \mathrm{~h}$. The solvent was evaporated to leave a black solid residue. The solid was dissolved in $20 \mathrm{~mL}$ of chloroform, washed with $3 \times 40 \mathrm{~mL}$ of aq $\mathrm{Na}_{2} \mathrm{CO}_{3}$, dried over anhydrous $\mathrm{MgSO}_{4}$ and evaporated under reduced pressure to give a yellow precipitate that was purified by column chromatography (silica, $95: 5$ dichloromethane:methanol) to yield $180 \mathrm{mg}(56 \%)$ of a light yellow powder with mp 224-226 ${ }^{\circ} \mathrm{C}$. HRMS(FAB, m/z): calcd for $\mathrm{C}_{68} \mathrm{H}_{58} \mathrm{O}_{10} 1034.3668$, found 1034.4030. ${ }^{1} \mathrm{H}-\mathrm{NMR}$ $\left(\mathrm{CDCl}_{3}\right): \delta 2.40(\mathrm{~m}, 4 \mathrm{H}), 2.82(\mathrm{t}, 4 \mathrm{H}, \mathrm{J}=7.2 \mathrm{~Hz}), 3.53(\mathrm{t}, 4 \mathrm{H}, \mathrm{J}=8 \mathrm{~Hz}), 3.67(\mathrm{~s}, 12 \mathrm{H}), 3.78(\mathrm{~s}, 7 \mathrm{H})$, $6.61(\mathrm{~s}, 4 \mathrm{H}), 6.95(\mathrm{~d}, 2 \mathrm{H}, \mathrm{J}=16 \mathrm{~Hz}), 7.01(\mathrm{~d}, 2 \mathrm{H}, \mathrm{J}=16 \mathrm{~Hz}), 7.42(\mathrm{~s}, 2 \mathrm{H}), 7.92-8.12(\mathrm{~m}, 20 \mathrm{H}) ;{ }^{13} \mathrm{C}-$ NMR $\left(\mathrm{CDCl}_{3}\right)$ : 26.98, 32.84, 33.97, 55.93, 60.95, 103.88, 120.21, 120.45, 123.18, 124.90, 125.10, 125.97, 126.93, 127.35, 127.44, 127.68, 128.75, 130.14, 130.83, 131.43, 131.87, 132.70, 135.02, 138.40, 145.89, 153.38, 171.54. UV-vis (THF, $\lambda_{\max } / \mathrm{nm}[\varepsilon]$ ): $243[127000], 265[57500], 277[74400], 328[62000]$, 344[82000], 393[42800].

$E, E-2,5-B i s($ undecanoloxy)-1,4-bis(3,4,5-trimethoxystyryl)benzene (19). In a round bottom flask a mixture of 18 (30 mg, $0.06 \mathrm{mmol})$, undecanoic acid (34 $\mathrm{mg}, 0.18 \mathrm{mmol})$, triphenylphospine (48 $\mathrm{mg}, 0.18 \mathrm{mmol})$, triethylamine $(0.03 \mathrm{~mL}, 0.18 \mathrm{mmol})$ and $1 \mathrm{~mL}$ of carbon tetrachloride in $15 \mathrm{~mL}$ of acetonitrile was stirred at room temperature for $24 \mathrm{~h}$. The solvent was evaporated to leave a black solid, which was dissolved in $20 \mathrm{~mL}$ of chloroform, washed with $3 \times 40 \mathrm{~mL}$ of aq $\mathrm{Na}_{2} \mathrm{CO}_{3}$, dried over anhydrous $\mathrm{MgSO}_{4}$ and evaporated under reduced pressure to give $40 \mathrm{mg}(80 \%)$ of solid with $\mathrm{mp} 103-105{ }^{\circ} \mathrm{C}$. HRMS (FAB, m/z): calcd for $\mathrm{C}_{50} \mathrm{H}_{70} \mathrm{O}_{10} 830.4827$, found 830.4969. ${ }^{1} \mathrm{H}-\mathrm{NMR}\left(\mathrm{CDCl}_{3}\right)$ : $\delta 0.87(\mathrm{t}, 6 \mathrm{H}, \mathrm{J}=6 \mathrm{~Hz})$, $1.25(\mathrm{~m}, 24 \mathrm{H}), 1.43(\mathrm{~m}, 4 \mathrm{H}), 1.83(\mathrm{~m}, 4 \mathrm{H}), 2.64(\mathrm{t}, 4 \mathrm{H}, \mathrm{J}=7.6 \mathrm{~Hz}), 3.87(\mathrm{~s}, 8 \mathrm{H}), 3.89(\mathrm{~s}, 16 \mathrm{H}), 6.69$ $(\mathrm{s}, 4 \mathrm{H}), 6.94(\mathrm{~d}, 2 \mathrm{H}, \mathrm{J}=16 \mathrm{~Hz}), 7.00(\mathrm{~d}, 2 \mathrm{H}, \mathrm{J}=16 \mathrm{~Hz}), 7.37(\mathrm{~s}, 2 \mathrm{H}) ;{ }^{13} \mathrm{C}-\mathrm{NMR}\left(\mathrm{CDCl}_{3}\right): 14.12,22.68$, 25.15, 29.30, 29.90, 31.88, 34.47, 56.11, 61.00, 64.80, 103.87, 120.14, 124.56, 127.35, 132.72, 138.43, 145.82, 153.41, 172.04. UV-vis (THF, $\left.\lambda_{\max } / \mathrm{nm}[\varepsilon]\right): 356[24900]$. 